OPEN ACCESS

Check for updates

\title{
Outcomes at follow-up of negative colonoscopy in average risk population: systematic review and meta-analysis
}

\author{
Thomas Heisser, ${ }^{1,2}$ Le Peng, ${ }^{1,2}$ Korbinian Weigl, ${ }^{1,4}$ Michael Hoffmeister, ${ }^{1}$ Hermann Brenner ${ }^{1,3,4}$
}

${ }^{1}$ Division of Clinical

Epidemiology and Aging

Research, German Cancer

Research Center (DKFZ), 69120

Heidelberg, Germany

${ }^{2}$ Medical Faculty Heidelberg

University of Heidelberg,

Heidelberg, Germany

${ }^{3}$ Division of Preventive

Oncology, German Cancer

Research Center (DKFZ) and

National Center for Tumor

Diseases (NCT), Heidelberg,

Germany

${ }^{4}$ German Cancer Consortium

(DKTK), German Cancer

Research Center (DKFZ)

Heidelberg, Germany

Correspondence to: $\mathrm{H}$ Brenner

h.brenner@dkfz.de

(ORCID 0000-0002-6129-1572)

Additional material is published online only. To view please visit

the journal online.

Cite this as: BMJ 2019;367:16109 http://dx.doi.org/10.1136/bmj.l6109

Accepted: 9 October 2019

\author{
ABSTRACT \\ OBJECTIVE \\ To review and summarise the evidence on the \\ prevalence of colorectal adenomas and cancers at \\ a follow-up screening colonoscopy after negative \\ index colonoscopy, stratified by interval between \\ examinations and by sex.
}

DESIGN

Systematic review and meta-analysis of all available studies.

\section{DATA SOURCES}

PubMed, Web of Science, and Embase. Two

investigators independently extracted characteristics and results of identified studies and performed standardised quality ratings.

\section{ELIGIBILITY CRITERIA}

Studies assessing the outcome of a follow-up colonoscopy among participants at average risk for colorectal cancer with a negative previous colonoscopy (no adenomas).

RESULTS

28 studies were identified, including 22 cohort studies, five cross sectional studies, and one case-control study. Findings for an interval between colonoscopies of one to five, five to 10 , and more than 10 years were reported by 17,16 , and three studies, respectively. Summary estimates of prevalences of any neoplasm were $20.7 \%$ (95\% confidence interval $15.8 \%$ to $25.5 \%$ ), $23.0 \%$ ( $18.0 \%$ to $28.0 \%$ ), and $21.9 \%$ (14.9\% to $29.0 \%$ ) for one to five, five to 10 , and more than 10 years between colonoscopies. Corresponding summary estimates of prevalences of any advanced neoplasm were $2.8 \%(2.0 \%$ to $3.7 \%)$, $3.2 \%(2.2 \%$ to $4.1 \%)$, and $7.0 \%$ (5.3\% to $8.7 \%)$. Seven studies also reported findings stratified by sex.

\section{WHAT IS ALREADY KNOWN ON THIS TOPIC}

For people at average risk without precursors of colorectal cancer at screening colonoscopy, major American and European guidelines recommend a next screening colonoscopy after a 10 year interval

The evidence supporting this interval is limited, and guidelines mostly rely on administrative claims data or linkage to cancer registries

The actual outcomes of a follow-up screening colonoscopy are rarely referred to as the basis of screening recommendations

\section{WHAT THIS STUDY ADDS}

Although neoplasms were detected in more than $20 \%$ of participants even within one to five years of a negative screening colonoscopy, detection of advanced

neoplasms within 10 years was rare

Recommendations of 10 year intervals for colonoscopy screening may be adequate, and repeated screening within the first 10 years after a negative colonoscopy has little additional benefit
Summary estimates stratified by interval and sex were consistently higher for men than for women.

\section{CONCLUSIONS}

Although detection of any neoplasms was observed in more than $20 \%$ of participants within five years of a negative screening colonoscopy, detection of advanced neoplasms within 10 years was rare. Our findings suggest that 10 year intervals for colonoscopy screening after a negative colonoscopy, as currently recommended, may be adequate, but more studies are needed to strengthen the empirical basis for pertinent recommendations and to investigate even longer intervals.

STUDY REGISTRATION

Prospero CRD42019127842.

\section{Introduction}

Colonoscopy is considered a powerful tool for reducing the incidence of and mortality from colorectal cancer and is recommended as a primary screening test by major medical authorities worldwide. ${ }^{1}$ For the general population at average risk-that is, people with no personal history of colorectal cancer, adenomas, or inflammatory bowel disease, no hereditary colorectal cancer syndrome, and no family history of colorectal cancer-major American and European guidelines recommend colonoscopic screening at 10 year intervals. ${ }^{1}$

However, this interval is mostly justified either by the assumed natural history of progression of adenomas into carcinomas or by anecdotal evidence relying on administrative claims data or linkage to cancer incidence registries. The actual outcomes of a followup screening colonoscopy, which should be paramount in defining a re-screening interval, are rarely referred to as a basis for screening recommendations. For instance, the recommendation of the US Preventive Services Task Force guidelines is based solely on a study with mortality as the endpoint, reporting incident cancers and deaths from colorectal cancer. ${ }^{2}$ The German Guideline Program in Oncology cites one small repeat colonoscopy study from 1996 with a mean interval between examinations of 5.5 years, ${ }^{3}$ as well as further indirect evidence from case-control studies, in which cases were composed of only participants with colorectal cancer. ${ }^{45}$ Moreover, despite these guideline recommendations, several studies have suggested considerable overuse of repeat screening colonoscopy before the end of the recommended interval. ${ }^{6-10}$

Comprehensive, high quality evidence on the prevalence of findings at repeat screening colonoscopies is needed to substantiate and possibly refine guideline recommendations, as well as to strengthen the efficiency of screening practice by decreasing overuse. We reviewed and summarised the available evidence 
from studies on the prevalence of findings at followup colonoscopies conducted at various time intervals after negative colonoscopy in people at average risk for colorectal cancer.

\section{Methods}

We carried out a systematic literature review and metaanalysis according to a predefined protocol (PROSPERO register: CRD42019127842). Reporting follows the PRISMA and MOOSE statements. ${ }^{11} 12$

\section{Data sources and searches}

We searched the electronic databases of PubMed, Web of Science, and Embase for eligible studies from inception to 14 June 2019. Search terms and their combinations used in the search strategy included colon, neoplasm, adenoma, polyp, follow-up, repeat, colonoscopy, and endoscopic examination. The search strategy is detailed in supplementary appendix 1. We searched the reference lists of identified publications for additional relevant studies and also scanned reference lists of related articles and guidelines.

\section{Study selection}

Published studies were eligible for inclusion if they assessed the outcome of a follow-up colonoscopy in participants at average risk for colorectal cancer with a negative previous colonoscopy (no adenomas). The target population consisted of people who were asymptomatic at presentation for the follow-up colonoscopy with "screening" as the only indication. However, mixed populations, including people with family history or an indication for colonoscopy due to symptoms at the time of the follow-up colonoscopy, were also eligible. Studies assessing tandem, back-toback, or early repeat colonoscopies were not eligible. The review was restricted to original articles published in the English language. The first author screened full text articles for eligibility, and ambiguities were resolved by discussion among all authors.

\section{Data extraction and quality assessment}

Two reviewers (TH and LP) independently used a standardised form to extract the following data: first author, year of publication, country/region, study type and setting, number and age of participants, interval between index and follow-up colonoscopies, and the proportion of participants with a family history, white ethnicity, and male sex. In addition, we extracted statements on the indication for colonoscopy to qualitatively assess the proportion of participants at average risk with screening as the indication for colonoscopy.

We extracted the prevalences of the most advanced findings at follow-up colonoscopy as far as reported. The main outcome parameters were the prevalence of any neoplasm and the prevalence of any advanced neoplasm. The former included any neoplasms (adenomas and cancers), and the latter included advanced adenomas and carcinomas. We defined an advanced adenoma as at least one polyp with any of the following characteristics: greater than $9 \mathrm{~mm}$ in size, villous or tubulovillous histology, or high grade dysplasia. ${ }^{1314}$ If studies differed from these definitions in their classification, we used appropriate surrogates to ensure comparability (supplementary table A).

We did a quality assessment of included studies for descriptive purposes. We used a modified version of the Newcastle-Ottawa Scale for cohort studies. ${ }^{15}$ We modified the scale in a way that allowed us to evaluate a comprehensive set of qualitative aspects relevant to answering the research question. For instance, we dropped the pre-specified question on the selection of non-exposed individuals (not applicable for our study) and instead included a question assessing the representativeness of the study population regarding the proportion of participants with screening as the single indication for follow-up colonoscopy. Two reviewers (TH, LP) independently assessed study quality and resolved disagreements by further review and discussion.

\section{Data synthesis and analysis}

We combined the studies in a narrative synthesis, focusing on differences in prevalences of any neoplasm and of any advanced neoplasm according to different intervals between the colonoscopies and the proportion of participants with screening as the indication for colonoscopy, as well as by sex. We categorised the time interval between index and followup colonoscopies as one to five years, five to 10 years, and more than 10 years. Wherever possible, we used the reported interval range; otherwise, we categorised the studies according to the mean interval. For studies reporting subgroups stratified for interval between colonoscopies, we included only the subgroups, and not the overall study population, in the quantitative and qualitative synthesis to avoid double counting.

We assessed heterogeneity in prevalence estimates by using $\mathrm{I}^{2}, \tau^{2}$, and Cochran's $\mathrm{Q}$ statistic, ${ }^{16}$ and we calculated summary prevalence estimates for the prevalence of any neoplasm and the prevalence of any advanced neoplasm together with 95\% confidence intervals, stratified by interval between procedures, proportion of participants with screening colonoscopy indication, and sex. If no heterogeneity was present ( $>>0.05)$, we calculated summary prevalence estimates by using fixed effects models. In the case of significant heterogeneity $(\mathrm{P} \leq 0.05)$, we used random effects models. ${ }^{17}$ We assessed publication bias with funnel plots. ${ }^{18}$ We used the "metafor" package in $\mathrm{R}$ version 3.5.0 to do meta-analyses. ${ }^{19}$

\section{Patient and public involvement}

Patients and the public were not involved in the design and conduct of this systematic review, which focuses on published literature. Patients were not invited to contribute to the writing or editing of this document for readability or accuracy. Research at the German Cancer Research Center (DKFZ) is generally informed by a Patient Advisory Committee. 


\section{Results}

\section{Study selection}

Figure 1 shows a flowchart of the study selection process. The search in PubMed, Web of Science, and Embase resulted in 10811 records. After exclusion of duplicates $(\mathrm{n}=4420)$ and removal of records not suitable for inclusion after screening of the title $(n=5452)$ or abstract $(n=821), 118$ articles were eligible for further assessment. For one study, the full text was not available in English. At full text review, we excluded 34 studies that assessed an ineligible study population; most of these included participants with a history of adenoma. We excluded another 17 studies because they assessed follow-up sigmoidoscopies rather than colonoscopies. Furthermore, we excluded 34 studies that did not assess the outcome of interest, such as the incidence of carcinomas or the use of or adherence to follow-up colonoscopies only. Finally, we excluded five papers because they were guidelines, reviews, or editorials. In total, we included 28 articles, ${ }^{3820-43}$ one of which we found via cross referencing. ${ }^{33}$

\section{Study characteristics}

\section{Overall study description}

Supplementary table B gives an overview of design aspects of the included studies, all of which assessed the prevalence of findings at a follow-up colonoscopy after a negative colonoscopy. Fifteen studies were from the Western hemisphere, ${ }^{38202123252930323335-39}$ and 13 were from the Eastern hemisphere. ${ }^{22} 2426-28313440-424445$ Twenty two studies were cohort studies, ${ }^{3} 820222425$ 27-32 $3437-45$ and five were cross sectional studies. ${ }^{21} 23263536$ One study was a casecontrol study in which the control group was prospectively followed up. ${ }^{33}$

The number of participants ranged from 29 in the (to our knowledge) first study to be published on this topic in 1994 to 15719 in a large retrospective analysis using the US. National Endoscopic Database published in 2014. ${ }^{29} 37$ The assessed time period across all studies, where reported, covers more than 30 years, ranging from 1986 to $2017 .^{3345}$

The mean age at follow-up colonoscopy ranged from 40 to 68 years, ${ }^{2023}$ and the proportion of men ranged from $42 \%$ to close to $100 \%$ in three Veteran Affairs Medical Centre studies. ${ }^{20} 21323537$ A fourth study exclusively in Veteran Affairs data can be assumed to be likewise mainly composed of men. ${ }^{30}$ The shortest mean interval length between examinations of the included studies was 2.1 years, and the longest was 11.9 years. $^{2134}$

Quality criteria ratings are shown in supplementary table C. All but two studies reported adequately that follow-up colonoscopy outcomes were ascertained on the basis of medical records. ${ }^{35}$ Most studies $(20 / 28)$ were rated as including representative participants for the general population at average risk. The proportion of participants with index and follow-up colonoscopies, compared with participants with only a first colonoscopy, was generally low. Only in seven studies did more than $50 \%$ of participants with negative index colonoscopic examination also attend a follow-up colonoscopy. Apart from this, lack of representativeness for a population with screening as the indication for follow-up colonoscopy was the most commonly identified qualitative limitation. Only 16 studies fulfilled this criterion.

\section{Subgroup analyses}

From 14 of the 28 identified studies, data from subgroup analyses were available. Seven articles reported findings stratified according to different intervals between the procedures, ${ }^{21} 262729323640$ and another seven articles reported findings stratified by sex. $^{3252633353642}$

\section{Findings}

Interval between negative index and follow-up colonoscopy

Figure 2 shows a forest plot of the prevalences of any neoplasm, prevalences of any advanced neoplasm, and meta-analysis summary estimates of all included studies. Findings for an interval between colonoscopies of one to five, five to 10 , and more than 10 years were reported by 17,16 , and three studies, respectively.

One to five years-The prevalences of any neoplasm varied substantially across the studies, ranging from $4.9 \%$ to $40.4 \%$; $50 \%$ of the observations were between $15.6 \%$ and $24.5 \%$, with a median prevalence of $21.4 \%$. The reported prevalences of any advanced neoplasm were less heterogeneous and ranged from $0.7 \%$ to $7.0 \%$, with $50 \%$ of the values being below $2.8 \%$ (interquartile range $2.1-4.3 \%$ ). For 13 of the studies, the prevalences of cancers could be derived, which were consistently low. In six studies, no cases with cancer were found, and another six studies reported prevalences below $0.8 \%$. The highest reported prevalence was $1.1 \%{ }^{32}$ (supplementary table D). The summary estimates were $20.7 \%$ (95\% confidence interval $15.8 \%$ to $25.5 \%$ ) for the prevalence of any neoplasm and $2.8 \%(2.0 \%$ to $3.7 \%$ ) for the prevalence of any advanced neoplasm, suggesting a low risk of advanced findings within five years of a negative index colonoscopy. Estimates were slightly lower when we limited the analyses to studies in which most participants had a screening indication (fig 3).

Five to 10 years-Reported prevalences of any neoplasm ranged from $7.4 \%$ to $41.4 \%$, with a median of $22.4 \%$ (interquartile range 20.4-27.2\%). Prevalences of any neoplasm ranged from $0.6 \%$ to $9.4 \%$, with a median of 3.6\% (1.6-5.0\%). Reported prevalences of cancers were generally low. In 10 studies, no cancers were found, and in three studies prevalences up to $0.5 \%$ were reported. The study by Matsuda and colleagues reported an exceptionally high cancer prevalence of $2.2 \%$. ${ }^{31}$ With $23.0 \%$ (95\% confidence interval $18.0 \%$ to $28.0 \%$ ) and $3.2 \%$ (2.2\% to $4.1 \%)$ for the prevalence of any neoplasm and prevalence of any advanced neoplasm, respectively, the summary estimates and 95\% confidence intervals were comparable to those for participants who had a follow-up colonoscopy after one to five years. The summary estimate for the 


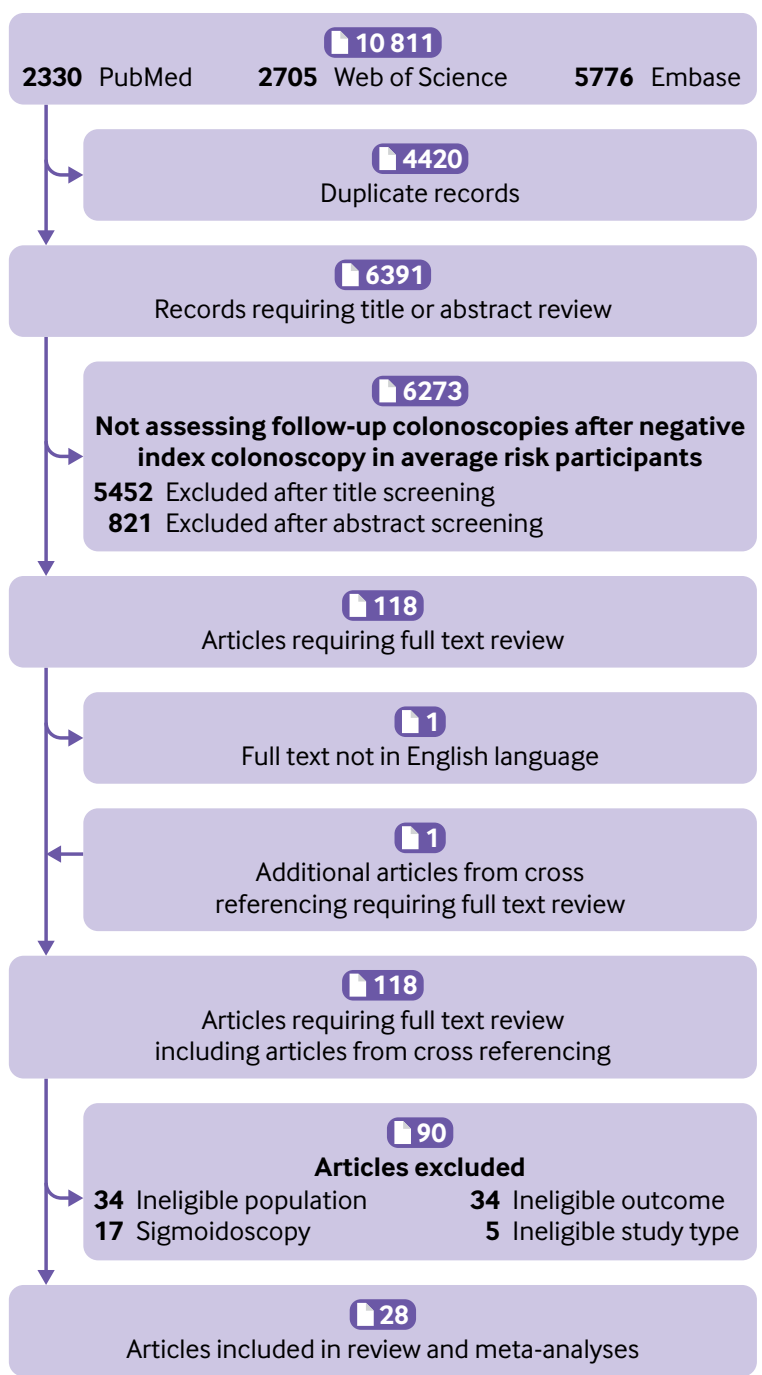

Fig 1 | Flowchart of literature search process

prevalence of any neoplasm was again slightly lower when we restricted the analyses to studies in which most participants had a screening indication at repeat colonoscopy.

More than 10 years-Only three studies reported results for more than 10 years after negative colonoscopy. ${ }^{21} 2736$ Prevalences of any neoplasm were $15.8 \%, 27.8 \%$, and $22.2 \%$; corresponding prevalences of any advanced neoplasms were $7.5 \%$, $8.0 \%$, and $5.6 \%$. In the study by Laish et al, ${ }^{27}$ cancer was detected in $2.4 \%$ of patients. No cancers were found in the other two studies. The summary estimate for the prevalence of any neoplasm, at $21.9 \%$ (95\% confidence interval $14.9 \%$ to $29.0 \%$ ), was comparable to those for participants with shorter intervals between colonoscopies. In contrast, at $7.0 \%$ (5.3\% to $8.7 \%$ ), the summary estimate for the prevalence of any advanced neoplasm was approximately twice as high as the summary estimates after one to five years $(2.8 \%)$ and five to 10 years (3.2\%). Limiting the analyses to study populations with a screening indication at follow-up colonoscopy for most participants did not particularly affect the summary estimates.

\section{Sex specific analyses}

Seven studies reported prevalences stratified by sex. In all but one study, ${ }^{33}$ the prevalence of any neoplasm was higher for men than for women. None of the individual studies suggested a statistically significant difference between the sexes in terms of detected advanced neoplasms.

For the meta-analyses, we included four studies in Veteran Affairs centres that were almost exclusively conducted in men. Summary estimates of the prevalence of any neoplasm and the prevalence of any advanced neoplasm stratified by interval and sex were consistently higher for men than for women (fig 4), suggesting a tendency towards more (advanced) neoplasms in men. However, owing to the limited number of studies, the calculated estimates for women were highly uncertain, limiting the potential for testing for statistical significance and for comparing the magnitude of differences between the prevalences.

\section{Heterogeneity between studies and risk of bias}

High levels of heterogeneity between the studies were evident in the interval groups of one to five and five to 10 years (supplementary table E1). On visual inspection of the funnel plots, prevalences of any advanced neoplasm seem markedly less heterogeneous and more evenly distributed than prevalences of any neoplasm (supplementary figures A and B). Taking into account that prevalences below $0 \%$ are not possible, the funnel plots did not suggest relevant reporting bias.

We did sensitivity analyses for ethnicity to assess whether and to what extent the heterogeneity could be removed (supplementary tables E2 and E3). Even though high levels of heterogeneity generally persisted, the summary estimates were robust.

For the analyses by sex, heterogeneity between the studies was moderate to high for both any neoplasms and any advanced neoplasms and was slightly less pronounced for male than for female subgroups. Funnel plots showed several outliers but overall did not suggest the presence of reporting bias (supplementary figure C).

\section{Discussion}

This systematic review and meta-analysis underpins the existing guideline recommendations to repeat screening no earlier than 10 years after a negative colonoscopy in people at average risk for colorectal cancer. Of the identified studies, the meta-analysis summary estimates for the prevalences of any neoplasm were on a comparable level (21\% to 23\%) for the three pre-defined time intervals. Whereas summary estimates of the prevalences of any advanced neoplasm (which were almost exclusively advanced adenomas) were $2.8 \%$ and $3.2 \%$ for intervals from one to five and five to 10 years after index colonoscopy, respectively, the estimate for the interval beyond 10 years was more than twice as high (7.0\%). Heterogeneity between the studies was substantial ( $\mathrm{I}^{2}>85 \%$ in five out of six meta-analyses); however, for all time intervals, extracted median values were in line with the summary estimates. 
RESEARCH

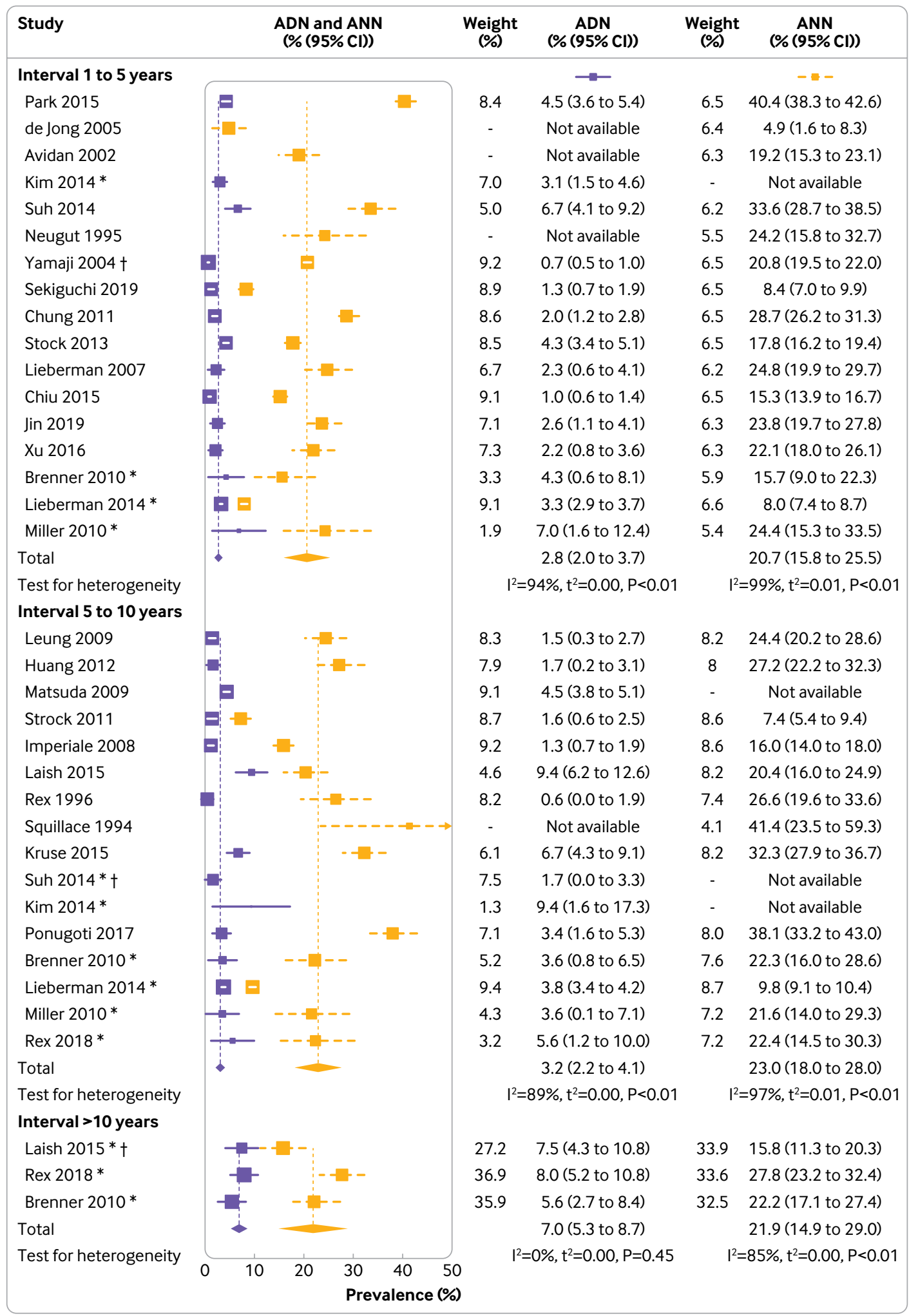

Fig 2 | Findings at follow-up colonoscopy, stratified by interval since negative colonoscopy. ADN=prevalence of any advanced neoplasm; $\mathrm{ANN}=$ prevalence of any neoplasm. *Interval subgroup. + Patients with two previously negative colonoscopies. $P$ values are based on Cochran's $Q$ statistic

Findings in context

The summary estimates and the median values of the prevalence of any neoplasm in our study are generally comparable to reported prevalences for first time screening. ${ }^{46-48}$ Given that study populations were pre-selected as being free of neoplasms at index colonoscopy, this seems unexpectedly high. With estimated annual rates of adenoma development of approximately $2 \%,{ }^{49}$ a large proportion of these adenomas may have been developed de novo since the index colonoscopy. Interestingly, however, our analyses did not suggest a pronounced time trend of 


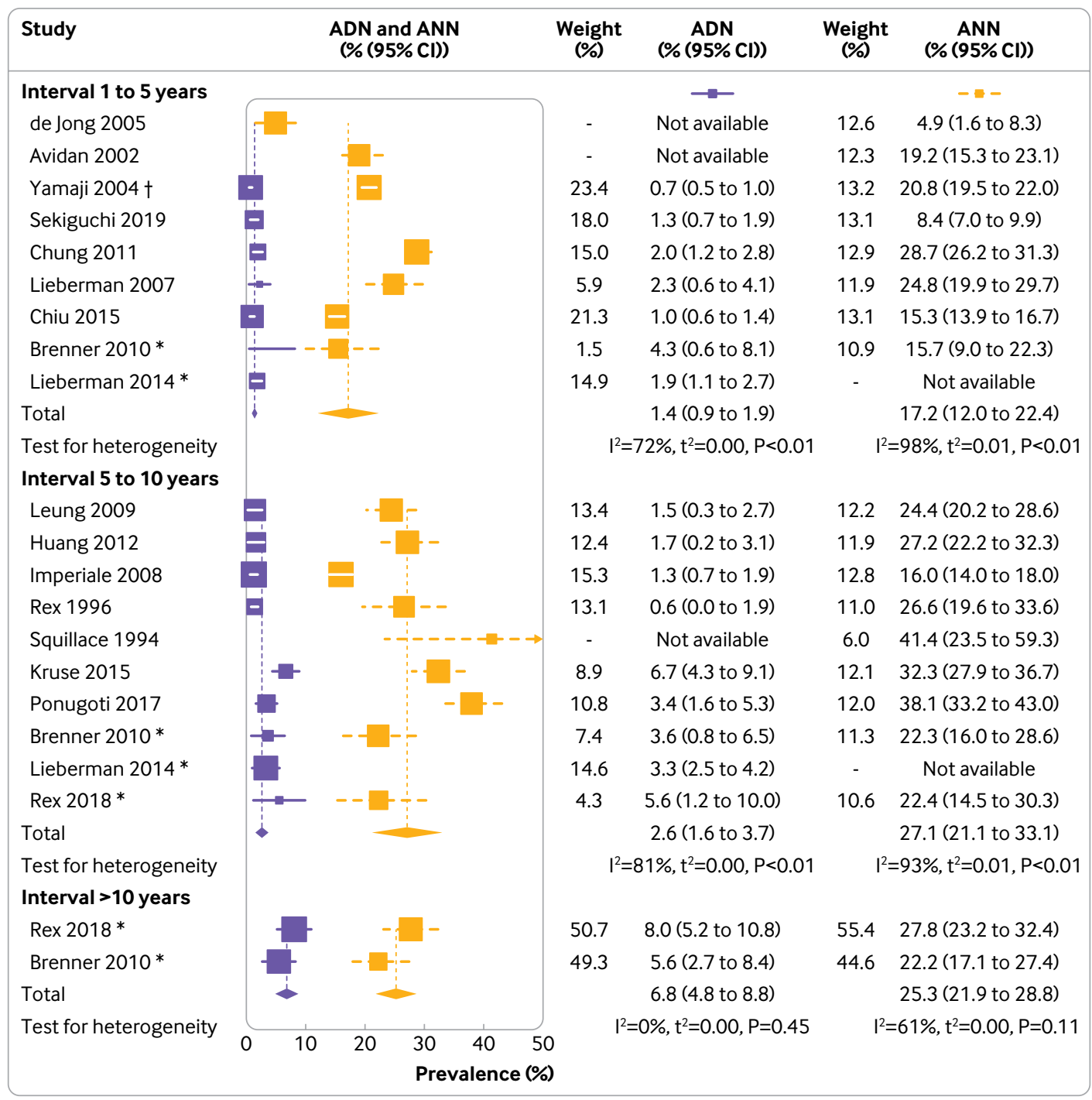

Fig 3 | Findings at follow-up colonoscopy for studies in which most participants had screening as indication for follow-up colonoscopy, stratified by interval since negative colonoscopy. ADN=prevalence of any advanced neoplasm; $\mathrm{ANN}=$ prevalence of any neoplasm. *Interval subgroup. + Patients with two previously negative colonoscopies. P values are based on Cochran's Q statistic

longer intervals expected to be associated with higher prevalences of any neoplasm.

Both findings-the seemingly high prevalence of any neoplasm at short intervals between colonoscopies and the limited increase in prevalence with longer intervals-may partly be explained by miss rates of any neoplasm. For example, assuming a true prevalence of any neoplasm of $30 \%$ and a $25 \%$ miss rate at index colonoscopy, ${ }^{50}$ one would expect that the $77.5 \%$ apparently neoplasm-free participants at index colonoscopy includes $70 \%$ truly neoplasmfree people and $7.5 \%(25 \% \times 30 \%)$ people with neoplasms misclassified as being neoplasm-free. At follow-up colonoscopies, when the missed adenomas are likely to have grown and thus become more easily detectable, one would expect to detect a prevalence of any neoplasm of approximately 10\% (7.5\%/77.5\%) even in the absence of any newly developed adenomas, regardless of the timing of the follow-up colonoscopy. This might explain a large proportion of the high prevalence of any neoplasm already seen one to five years after the index colonoscopy.

Furthermore, people with poorer bowel preparation at index colonoscopy, who are expected to have higher miss rates, may be more likely to be recommended to undergo follow-up colonoscopies after shorter time intervals than people with better bowel preparation. The same may apply to people deemed to be at higher risk of developing colorectal neoplasms for other reasons, such as lifestyle related risk factors. ${ }^{51}$ The potential over-representation of such people at higher risk in the short interval group compared with the longer interval groups may also contribute to both the high prevalence of any neoplasm in the interval group of one to five years and the apparently modest increase in prevalence with increasing length of intervals between colonoscopies. Nevertheless, despite these likely selection processes, the increase in prevalence of any neoplasm from $17.2 \%$ to $27.1 \%$ between the one to five year and the five to 10 year interval groups in 


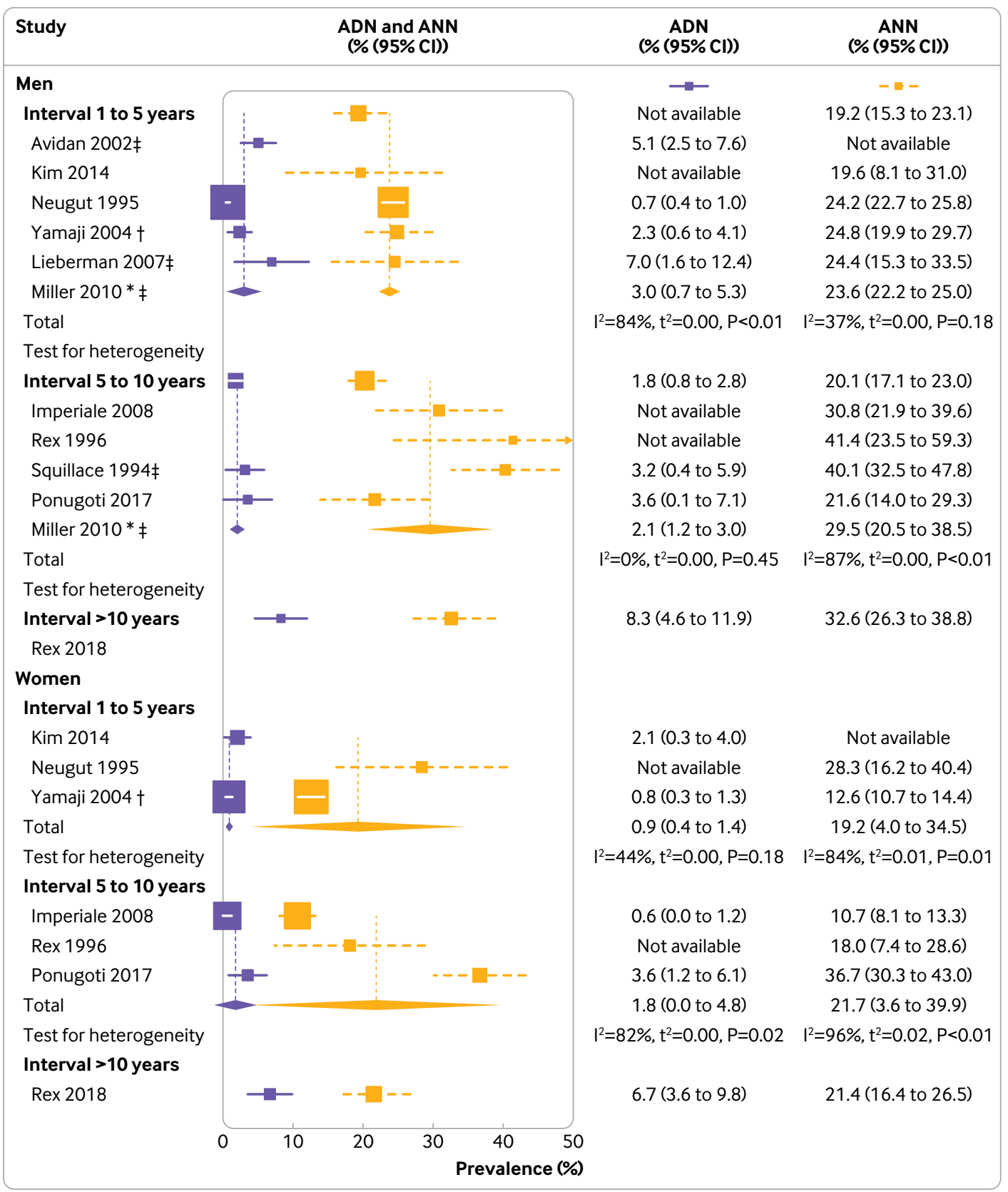

Fig 4 | Findings at follow-up colonoscopy among men and women, stratified by interval since negative colonoscopy. $A D N=$ prevalence of any advanced neoplasm; $A N N=$ prevalence of any neoplasm. *Interval sub-cohort. +Cohort with two previously negative colonoscopies. ¥Cohort of male Veteran Affairs. P values are based on Cochran's Q statistic

the studies in which most participants had screening as an indication are compatible with the assumed $2 \%$ incidence per year.

The estimated yield of approximately $3 \%$ of advanced neoplasms and the very low prevalences of cancer over the first 10 years constitute evidence encouraging people without symptoms who are at average risk, as well as their physicians, to adhere to the full recommended 10 years between the colonoscopies. Moreover, the annual transition rate from nonadvanced to advanced adenomas was previously estimated to be around $4 \%,{ }^{52}$ which suggests that most of the advanced lesions detected at follow-up did not develop de novo but were probably missed at index colonoscopies. Some of the advanced neoplasms detected may also be related to biological factors leading to rapid progression. ${ }^{53}$ Incomplete removal or accelerated polyp formation may also contribute to explaining the substantial increase in the prevalence of advanced neoplasms beyond 10 years. However, given that the summary estimate (and median value) for prevalence of any advanced neoplasm was more than doubled, thereby reaching a prevalence similar to first time screening, ${ }^{46-48}$ it is plausible to assume that a part of these findings truly represents new lesions that developed via the adenomatous polyps pathway. ${ }^{54}$

Importantly, the evidence should not be overinterpreted by inferring a fixed threshold of 10 
years after which a higher prevalence of advanced neoplasms immanently manifests. Firstly, the evidence beyond 10 years was limited to only three studies, and, secondly, two of these also included participants with an interval beyond 15 years. Therefore, uncertainty remains as to whether and for how long the interval between procedures can be safely extended beyond 10 years. Beyond 10 years, cancers were found only in the study by Laish and colleagues. ${ }^{27}$ The generalisability of these findings should be interpreted with caution, however, given the study's low quality rating. More high quality studies with intervals of at least 10 years between examinations are needed to substantiate and refine our findings.

Finally, the proportions of adenomas missed under standardised conditions in study settings, which may in particular apply to the prospective studies included in our review, 32224283033374244 could be substantially lower than in routine practice in the community setting. ${ }^{50556}$ In line with that, another study published in the BMJ found a significantly lower incidence of post-colonoscopy colorectal cancer (rate of cancers detected within six to 36 months after a negative index colonoscopy divided by all cancers detected within 36 months) in screening centres where colonoscopists underwent an accreditation process involving strict, standardised performance targets (caecal intubation rate $>92 \%$ and adenoma detection rate $>40 \%) .{ }^{57}$ Although our review supports full adherence to the recommended 10 year interval, the fact that a high procedural quality of the index colonoscopy remains a paramount prerequisite should be kept in mind.

As we expected the body of evidence to be limited, we chose an approach that also allowed mixed study populations including participants with indications other than screening. We deemed this the best strategy to increase the power and robustness of the main analyses, as the symptoms and signs of adenomas and cancer are non-specific. ${ }^{58}$ Only between about a fifth and a third of participants with symptoms referred for colonoscopy actually have significant colorectal disease, and even fewer will have colorectal neoplasms. ${ }^{59}$ In addition, the 10 year interval is generally recommended after a negative colonoscopy for people at average risk, regardless of the indication for the index colonoscopy. ${ }^{1}$ Thus, it is not surprising that when we restricted the analyses to the subset in which most participants had a screening indication at follow-up colonoscopy, the summary estimates were overall in accordance with the estimates for all studies.

Our review suggests higher prevalences of any neoplasm and of any advanced neoplasms in men than in women. This is in agreement with prevalences reported from first time screening studies, ${ }^{46-48}$ as well as the evidence on the higher risk of colorectal cancer in men, leading to an elevated incidence of colorectal cancer in men. ${ }^{60}$ Unfortunately, the limited evidence, in particular for women, prohibits the drawing of conclusions regarding the magnitude of differences in findings. More studies with larger sample sizes and sex specific analyses are needed to allow judgment of potential use and relevance of recommendations for sex specific screening interval.

\section{Comparison with other studies}

To our knowledge, ours is the first systematic review and meta-analysis on findings at a followup colonoscopy after a negative colonoscopy. The closest comparative data would be from studies of cancer incidence in people without findings at first time screening colonoscopy. The trend to a low risk for advanced neoplasms within the first 10 years seen in our review, and even beyond 10 years in terms of cancers, is consistent with what has been reported for several cohort and case-control studies assessing cancer incidence. ${ }^{25}$ 61-63 Notably, several of these studies found that the low risk of cancer was sustained for periods exceeding 10 years by far, suggesting that it might even last for 15 or 20 years.

\section{Limitations of this review}

Our review and meta-analysis has several limitations. Firstly, it is entirely based on observational studies, which may be affected by confounding factors associated with use of colonoscopy. In addition, the proportion of participants with index and follow-up colonoscopies, compared with those with only a first colonoscopy, was generally low. This suggests potential for selection bias, as participants with repeated examinations either might be more health conscious or may have had a diagnostic reason to return. However, willingness to undergo screening is unlikely to be a predictor of neoplastic findings, ${ }^{64}$ and the summary estimates including mixed study populations deviated only slightly from those for participants at average risk.

Secondly, a considerable proportion of studies in our review also included participants with a family history of colorectal cancer, for whom some guidelines recommend shorter intervals as they might still be at higher risk for colorectal cancer. ${ }^{65}$ However, their inclusion suggests that the true prevalences for participants at average risk might even be lower.

Thirdly, considerable heterogeneity existed in the design of the included studies. As a result, the statistical heterogeneity in the meta-analyses was substantial and could not be significantly reduced in sensitivity analyses. Even if categorised per interval, a variety of factors will contribute to intra-group heterogeneity, including population characteristics and factors outside the scope of this study, such as the skills of individual endoscopists.

\section{Implications for colorectal cancer screening}

Although any neoplasms were detected in more than $20 \%$ of participants even at colonoscopies conducted within five years of a negative colonoscopy, detection of advanced neoplasms within 10 years was rare. This finding suggests that recommendations of 10 year intervals for colonoscopy screening in this low risk group may be adequate. As the large majority of nonadvanced lesions are commonly thought to have a very low potential for transition to cancer, the high median 
values for detection of any neoplasms should not be regarded as a rationale to repeat screening earlier than after 10 years.

As previously noted, ${ }^{21}$ the long lasting lower risk of advanced colorectal neoplasia after a negative colonoscopy is not to be interpreted as a protective effect of colonoscopy because no polyps were removed. Rather, our study corroborates the notion that participants who were found to be free of polyps at an index colonoscopy at screening age have an inherently lower risk. $^{2136}$

\section{Conclusion}

Even though this systematic review and meta-analysis was solely based on observational studies, as, to our knowledge, no randomised controlled trials on this topic exist, ${ }^{66}$ it provides the highest level of evidence currently possible on outcomes of a follow-up screening colonoscopy in people at average risk after a negative index colonoscopy.

Our study showed that detection of advanced neoplasms within 10 years is rare. This suggests that recommendations of 10 year intervals for colonoscopy screening may be adequate and indicates little additional benefit of repeated screening within the first 10 years of a negative index colonoscopy.

Uncertainty remains about a potential expansion of the 10 year period and the magnitude of potential differences between men and women. Future studies should strengthen the empirical basis for pertinent recommendations, focus on ranges exceeding 10 years between examinations, and report findings stratified by sex.

Contributors: HB and TH designed the study. TH did the literature search. TH and LP extracted the data from the identified articles. TH did the meta-analyses and drafted the manuscript. All authors critically reviewed the manuscript, contributed to its revision, and approved the final version submitted. All authors had full access to al of the data (including statistical reports and tables) in the study and can take responsibility for the integrity of the data and the accuracy of the data analysis. The corresponding author attests that all listed authors meet authorship criteria and that no others meeting the criteria have been omitted. HB is the guarantor.

Funding: This work was supported by the Ministry of Education and Research (grant number 01GL1712). The sponsor had no role in the study design; the collection, analysis, and interpretation of data; the writing of the report; or the decision to submit the article for publication. TH was supported by the Helmholtz International Graduate School for Cancer Research at the German Cancer Research Centre (DKFZ).

Competing interests: All authors have completed the ICMJE uniform disclosure form at www.icmje.org/coi_disclosure.pdf (available on request from the corresponding author) and declare: support from the German Research Council and the German Federal Ministry of Education and Research; no financial relationships with any organisations that might have an interest in the submitted work in the previous three years; no other relationships or activities that could appear to have influenced the submitted work.

Ethical approval: Not needed.

Data sharing: No additional data available.

Transparency: The corresponding author affirms that the manuscript is an honest, accurate, and transparent account of the study being reported; that no important aspects of the study have been omitted; and that any discrepancies from the study as planned (and, if relevant, registered) have been explained.

This is an Open Access article distributed in accordance with the Creative Commons Attribution Non Commercial (CC BY-NC 4.0) license, which permits others to distribute, remix, adapt, build upon this work non-commercially, and license their derivative works on different terms, provided the original work is properly cited and the use is noncommercial. See: http://creativecommons.org/licenses/by-nc/4.0/.

1 Bénard F, Barkun AN, Martel M, von Renteln D. Systematic review of colorectal cancer screening guidelines for average-risk adults: Summarizing the current global recommendations. World I Gastroenterol 2018;24:124-38. doi:10.3748/wjg.v24.i1.124

2 Nishihara R, Wu K, Lochhead P. Long-term colorectal-cancer incidence and mortality after lower endoscopy. N Engl J Med 2013;369:1095105. doi:10.1056/NEJMoa1301969

3 Rex DK, Cummings OW, Helper DJ. 5-year incidence of adenomas after negative colonoscopy in asymptomatic average-risk persons [see comment]. Gastroenterology 1996;111:1178-81. doi:10.1053/ gast.1996.v111.pm8898630

4 Singh H, Nugent Z, Mahmud SM, Demers AA, Bernstein CN. Predictors of colorectal cancer after negative colonoscopy: a populationbased study. Am J Gastroenterol 2010;105:663-73, quiz 674 doi:10.1038/ajg.2009.650

5 Brenner H, Chang-Claude J, Seiler CM, Hoffmeister M. Longterm risk of colorectal cancer after negative colonoscopy. J Clin Oncol 2011;29:3761-7. doi:10.1200/JCO.2011.35.9307

6 Hoffmeister M, Holleczek B, Stock C. Utilization and determinants of follow-up colonoscopies within 6 years after screening colonoscopy: Prospective cohort study. Int J Cancer 2019;144:402-10. doi:10.1002/ijc.31862

7 Murphy CC, Sandler RS, Grubber IM, Johnson MR, Fisher DA Underuse and Overuse of Colonoscopy for Repeat Screening and Surveillance in the Veterans Health Administration. Clin Gastroenterol Hepatol 2016;14:436-444.e1. doi:10.1016/j.cgh.2015.10.008

8 Kruse GR, Khan SM, Zaslavsky AM, Ayanian IZ, Sequist TD. Overuse of colonoscopy for colorectal cancer screening and surveillance. / Gen Intern Med 2015;30:277-83. doi:10.1007/s11606-014-3015-6

9 Schoen RE, Pinsky PF, Weissfeld JL. Utilization of surveillance colonoscopy in community practice. Gastroenterology 2010;138:73 81. doi:10.1053/j.gastro.2009.09.062

10 Goodwin IS, Singh A, Reddy N, Riall TS, Kuo YF. Overuse of screening colonoscopy in the Medicare population. Arch Intern Med 2011;171:1335-43. doi:10.1001/archinternmed.2011.212

11 Liberati A, Altman DG, TetzlaffJ. The PRISMA statement for reporting systematic reviews and meta-analyses of studies that evaluate healthcare interventions: explanation and elaboration. BMJ 2009;339:b2700. doi:10.1136/bmj.b2700

12 Stroup DF, Berlin JA, Morton SC. Meta-analysis of observational studies in epidemiology: a proposal for reporting. Meta-analysis Of Observational Studies in Epidemiology (MOOSE) group. JAMA 2000;283:2008-12. doi:10.1001/jama.283.15.2008

13 Arditi C, Gonvers IJ, Burnand BEPAGE II Study Group. Appropriateness of colonoscopy in Europe (EPAGE II). Surveillance after polypectomy and after resection of colorectal cancer. Endoscopy 2009;41:209-17. doi:10.1055/s-0028-1119646

14 Gimeno García AZ, González Y, Quintero E. Clinical validation of the European Panel on the Appropriateness of Gastrointestinal Endoscopy (EPAGE) II criteria in an open-access unit: a prospective study. Endoscopy 2012;44:32-7. doi:10.1055/s-0031-1291386

15 Wells GA, Shea B, O'Connell D, et al. The Newcastle-Ottawa Scale (NOS) for assessing the quality of nonrandomised studies in metaanalyses. 2013 www.ohri.ca/programs/clinical_epidemiology/ oxford.asp.

16 Higgins JP, Thompson SG. Quantifying heterogeneity in a metaanalysis. Stat Med 2002;21:1539-58. doi:10.1002/sim.1186

17 DerSimonian R, Laird N. Meta-analysis in clinical trials. Control Clin Trials 1986;7:177-88. doi:10.1016/0197-2456(86)90046-2

18 Egger M, Davey Smith G, Schneider M, Minder C. Bias in metaanalysis detected by a simple, graphical test. BMJ 1997;315:629-34. doi:10.1136/bmj.315.7109.629

19 Viechtbauer W. Conducting meta-analyses in R with the metafor package. J Stat Softw 2010;36:1-48. doi:10.18637/jss.v036.i03.

20 Avidan B, Sonnenberg A, Schnell TG, Leya J, Metz A, Sontag SJ. New occurrence and recurrence of neoplasms within 5 years of a screening colonoscopy. Am / Gastroenterol 2002;97:1524-9. doi:10.1111/j.1572-0241.2002.05801.x

21 Brenner H, Haug U, Arndt V, Stegmaier C, Altenhofen L, Hoffmeister M. Low risk of colorectal cancer and advanced adenomas more than 10 years after negative colonoscopy. Gastroenterology 2010;138:8706. doi:10.1053/j.gastro.2009.10.054

22 Chung SJ, Kim YS, Yang SY. Five-year risk for advanced colorectal neoplasia after initial colonoscopy according to the baseline risk stratification: a prospective study in 2452 asymptomatic Koreans. Gut 2011;60:1537-43. doi:10.1136/gut.2010.232876

23 de Jong AE, Morreau H, Nagengast FM. Prevalence of adenomas among young individuals at average risk for colorectal cancer. Am J Gastroenterol 2005;100:139-43. doi:10.1111/j.1572$0241.2005 .41000 x$ 
24 Huang Y, Li X, Wang Z, Su B. Five-year risk of colorectal neoplasia after normal baseline colonoscopy in asymptomatic Chinese Mongolian over 50 years of age. Int J Colorectal Dis 2012;27:1651-6. doi:10.1007/s00384-012-1516-5

25 Imperiale TF, Glowinski EA, Lin-Cooper C, Larkin GN, Rogge JD, Ransohoff DF. Five-year risk of colorectal neoplasia after negative screening colonoscopy. N Engl J Med 2008;359:1218-24. doi:10.1056/NEJMoa0803597

26 Kim HG, Jeon SR, Lee TH, Ko BM, Kim JO, Lee JS. Rescreening colonoscopy practice after a negative index colonoscopy in a clinical setting; cross-sectional study for interval and outcomes of rescreening colonoscopy. Hepatogastroenterology 2014;61:2266-71.

27 Laish I, Blechman I, Feingelernt H, Konikoff FM. Yield of second surveillance colonoscopy to predict adenomas with high-risk characteristics. Dig Liver Dis 2015;47:805-10. doi:10.1016/j. dld.2015.05.005

28 Leung WK, Lau JY, Suen BY. Repeat-screening colonoscopy 5 years after normal baseline-screening colonoscopy in average-risk Chinese: a prospective study. Am J Gastroenterol 2009;104:2028-34. doi:10.1038/ajg.2009.202

29 Lieberman DA, Holub JL, Morris CD, Logan J, Williams JL, Carney P. Low rate of large polyps ( $>9 \mathrm{~mm}$ ) within 10 years after an adequate baseline colonoscopy with no polyps. Gastroenterology 2014;147:343-50. doi:10.1053/j. gastro.2014.04.020

30 Lieberman DA, Weiss DG, Harford WV. Five-year colon surveillance after screening colonoscopy. Gastroenterology 2007;133:1077-85. doi:10.1053/j.gastro.2007.07.006

31 Matsuda T, Fujii T, Sano Y. Five-year incidence of advanced neoplasia after initial colonoscopy in Japan: a multicenter retrospective cohort study. Jpn J Clin Oncol 2009;39:435-42. doi:10.1093/jico/hyp047

32 Miller HL, Mukherjee R, Tian J, Nagar AB. Colonoscopy surveillance after polypectomy may be extended beyond five years. J Clin Gastroenterol 2010;44:e162-6. doi:10.1097/ MCG Ob013e3181e5cd22

33 Neugut Al, Jacobson JS, Ahsan H. Incidence and recurrence rates of colorectal adenomas: a prospective study. Gastroenterology 1995;108:402-8. doi:10.1016/00165085(95)90066-7

34 Park HW, Han S, Lee JY. Probability of high-risk colorectal neoplasm recurrence based on the results of two previous colonoscopies. Dig Dis Sci 2015;60:226-33. doi:10.1007/s10620-014-3334-9

35 Ponugoti PL, Rex DK. Yield of a second screening colonoscopy 10 years after an initial negative examination in average-risk individuals. Gastrointest Endosc 2017;85:221-4. doi:10.1016/j. gie.2016.05.024

36 Rex DK, Ponugoti PL, Johnson CS, Kittner L, Yanda RJ. Neoplasia at 10-year follow-up screening colonoscopy in a private U.S. practice: comparison of yield to first-time examinations. Gastrointest Endosc 2018;87:254-9. doi:10.1016/j.gie.2017.04.03

37 Squillace S, Berggreen P, Jaffe P. A normal initial colonoscopy after age 50 does not predict a polyp-free status for life. Am J Gastroenterol 1994;89:1156-9.

38 Stock C, Hoffmeister M, Birkner B, Brenner H. Performance of additional colonoscopies and yield of neoplasms within 3 years after screening colonoscopy: a historical cohort study. Endoscopy 2013;45:537-46. doi:10.1055/s-0032-1326485

39 Strock P, Mossong J, Scheiden R, Weber J, Heieck F, Kerschen A. Colorectal cancer incidence is low in patients following a colonoscopy. Dig Liver Dis 2011;43:899-904. doi:10.1016/j. dld.2011.05.020

40 Suh KH, Koo JS, Hyun JJ. Risk of adenomas with high-risk characteristics based on two previous colonoscopy. J Gastroenterol Hepatol 2014:29:1985-90. doi:10.1111/jgh.12650

41 Xu M, Wang S, Cao H. Low rate of advanced adenoma formation during a 5-year colonoscopy surveillance period after adequate polypectomy of non-advanced adenoma. Colorectal Dis 2016;18:179-86. doi:10.1111/codi.13148

42 Yamaji Y, Mitsushima T, Ikuma H. Incidence and recurrence rates of colorectal adenomas estimated by annually repeated colonoscopies on asymptomatic Japanese. Gut 2004;53:568-72. doi:10.1136/ gut.2003.026112

43 Sekiguchi M, Otake Y, Kakugawa Y. Incidence of Advanced Colorectal Neoplasia in Individuals With Untreated Diminutive Colorectal Adenomas Diagnosed by Magnifying Image-Enhanced Endoscopy. Am J Gastroenterol 2019;114:964-73. doi:10.14309/ ajg.0000000000000261

44 Chiu HM, Lee YC, Tu CH. Effects of metabolic syndrome and findings from baseline colonoscopies on occurrence of colorectal neoplasms. Clin Gastroenterol Hepatol 2015;13:1134-42.e8. doi:10.1016/j. cgh.2014.10.022

45 Jin $\mathrm{D}, \mathrm{Cao} \mathrm{H}$, Shah BK. Low detection rate of advanced neoplasia within 5 years after polypectomy of small serrated adenoma. Postgrad Med / 2019;95:187-92. doi:10.1136/ postgradmedj-2018-136285

46 Arditi C, Peytremann-Bridevaux I, Burnand BEPAGE II Study Group. Appropriateness of colonoscopy in Europe (EPAGE II) Screening for colorectal cancer. Endoscopy 2009;41:200-8. doi:10.1055/s-0028-1119626

47 Rex DK, Lehman GA, Ulbright TM. Colonic neoplasia in asymptomatic persons with negative fecal occult blood tests: influence of age, gender, and family history. Am J Gastroenterol 1993;88:825-31.

48 Imperiale TF, Wagner DR, Lin CY, Larkin GN, Rogge JD, Ransohoff DF. Risk of advanced proximal neoplasms in asymptomatic adults according to the distal colorectal findings. N EnglJ Med 2000;343:169-74. doi:10.1056/NEIM200007203430302

49 Brenner H, Altenhofen L, Stock C, Hoffmeister M. Incidence of colorectal adenomas: birth cohort analysis among 4.3 million participants of screening colonoscopy. Cancer Epidemiol Biomarkers Prev 2014;23:1920-7. doi:10.1158/1055-9965.EPI-14-0367

50 Zhao S, Wang S, Pan P. Magnitude, Risk Factors, and Factors Associated With Adenoma Miss Rate of Tandem Colonoscopy: A Systematic Review and Meta-analysis. Gastroenterology 2019;156:1661-1674.e11. doi:10.1053/j.gastro.2019.01.260

51 Brenner H, Kloor M, Pox CP. Colorectal cancer. Lancet 2014:383:1490-502. doi:10.1016/S0140-6736(13)61649-9

52 Brenner H, Altenhofen L, Stock C, Hoffmeister M. Expected longterm impact of the German screening colonoscopy programme on colorectal cancer prevention: analyses based on 4,407,971 screening colonoscopies. Eur J Cancer 2015;51:1346-53. doi:10.1016/j.ejca.2015.03.020

53 Samadder NJ, Curtin K, Tuohy TMF. Characteristics of missed or interval colorectal cancer and patient survival: a population-based study. Gastroenterology 2014;146:950-60. doi:10.1053/j. gastro.2014.01.013

54 Winawer SJ. Natural history of colorectal cancer. Am J Med 1999;106(1A):3S-6S, discussion 50S-1S. doi:10.1016/S0002 9343(98)00338-6

55 Bressler B, Paszat LF, Vinden C, Li C, He J, Rabeneck L. Colonoscopic miss rates for right-sided colon cancer: a population-based analysis. Gastroenterology 2004;127:452-6. doi:10.1053/j. gastro.2004.05.032

56 Robertson DJ, Greenberg ER, Beach M. Colorectal cancer in patients under close colonoscopic surveillance. Gastroenterology 2005;129:34-41. doi:10.1053/j.gastro.2005.05.012

57 Burr NE, Derbyshire E, Taylor J. Variation in post-colonoscopy colorectal cancer across colonoscopy providers in English National Health Service: population based cohort study. BMJ 2019;16090:16090.

58 Thompson MR, Perera R, Senapati A, Dodds S. Predictive value of common symptom combinations in diagnosing colorectal cancer. $\mathrm{Br}$ Surg 2007;94:1260-5. doi:10.1002/bjs.5826

59 Elias SG, Kok L, Witteman BJM. Published diagnostic models safely excluded colorectal cancer in an independent primary care validation study. J Clin Epidemiol 2017;82:149-157.e8. doi:10.1016/j. jclinepi.2016.09.014

60 Bray F, Ferlay J, Soerjomataram I, Siegel RL, Torre LA, Jemal A. Global cancer statistics 2018: GLOBOCAN estimates of incidence and mortality worldwide for 36 cancers in 185 countries. CA Cancer) Clin 2018:68:394-424. doi:10.3322/caac.21492

61 Singh H, Turner D, Xue L, Targownik LE, Bernstein CN. Risk of developing colorectal cancer following a negative colonoscopy examination: evidence for a 10-year interval between colonoscopies. IAMA 2006:295:2366-73 doi:10.1001/jama.295.20.2366

62 Lakoff J, Paszat LF, Saskin R, Rabeneck L. Risk of developing proxima versus distal colorectal cancer after a negative colonoscopy: a population-based study. Clin Gastroenterol Hepatol 2008;6:1117 21, quiz 1064. doi:10.1016/j.cgh.2008.05.016

63 Brenner H, Chang-Claude J, Seiler CM, Rickert A, Hoffmeister M. Protection from colorectal cancer after colonoscopy: a populationbased, case-control study. Ann Intern Med 2011;154:22-30. doi:10.7326/0003-4819-154-1-201101040-00004

64 Jensen CD, Doubeni CA, Quinn VP. Adjusting for patient demographics has minimal effects on rates of adenoma detection in a large, community-based setting. Clin Gastroenterol Hepatol 2015;13:73946. doi:10.1016/j.cgh.2014.10.020

65 Henrikson NB, Webber EM, Goddard KA. Family history and the natural history of colorectal cancer: systematic review. Genet Med 2015;17:702-12. doi:10.1038/gim.2014.188

66 Bretthauer M, Kaminski MF, Løberg MNordic-European Initiative on Colorectal Cancer (NordICC) Study Group. Population-Based Colonoscopy Screening for Colorectal Cancer: A Randomized Clinical Trial. JAMA Intern Med 2016;176:894-902. doi:10.1001/ jamainternmed.2016.0960

Supplementary materials

Web appendix: Visual summary 